\title{
PACKET SCHEDULING STUDY FOR HETEROGENEOUS TRAFFIC IN DOWNLINK 3GPP LTE SYSTEM
}

\author{
Roshanak Heidari, Farhana Afroz, Ramprasad Subramanian, Sinhlam Cong, \\ Kumbesan Sandrasegaran and Xiaoying Kong \\ Faculty of Engineering and Information Technology \\ Centre for Real Time Information Networks, University of Technology Sydney, Sydney, \\ Australia
}

\begin{abstract}
Long Term Evolution (LTE) network deploys Orthogonal Frequency Division Multiple Access (OFDMA) technology for downlink multi-carrier transmission. To meet the Quality of Service $(Q o S)$ requirements for LTE networks, packet scheduling has been employed. Packet scheduling determines when and how the user's packets are transmitted to the receiver. Therefore effective design of packet scheduling algorithm is an important discussion. The aims of packet scheduling are maximizing system throughput, guaranteeing fairness among users, andminimizing either or both PacketLoss Ratio (PLR)and packet delay. Inthis paper, the performance of two packet scheduling algorithms namely Log Maximum-Largest Weighted Delay First (LOG-MLWDF) and Max Delay Unit (MDU), developed for OFDM(Orthogonal Frequency Division Multiplexing)networks, has been investigated in LTE downlink networks, and acomparison of those algorithmswith a well-known scheduling algorithm namely Maximum-Largest Weighted Delay First(MLWDF) has been studied.The performance evaluation was in terms of system throughput, PLR and fairness index. This study was performed forboth real time (voice and video streaming)and non-real time (best effort)perspectives. Results show that for streaming flows,LOG-MLWDF shows best PLR performance among the considered scheduling schemes, and for best effort flows, it outperforms theother two algorithms in terms of packet delay and throughput.
\end{abstract}

\section{KEYWORDS}

Packetscheduling, LTE,DSA, PLR, throughput

\section{INTRODUCTION}

Long Term Evolution (LTE) is a development by the Third-Generation Partnership Project (3GPP) to meet the needs of theInternational Mobile Telecommunication Union (ITU). Some of its key features include increased data rate, a scalable bandwidth, increased coverage and capacity, and reduced latency that result in better Quality of Services (QoS) in communication. It employs OFDMAfor downlink transmission. LTE radio access network consists of eNodeBs, which are responsible for radio resource management function including packet scheduling. Packet scheduling is responsible for smart selection of user packetsand allocation radio resources 
International Journal of Wireless \& Mobile Networks (IJWMN) Vol. 7, No. 5, October 2015

appropriately. There are two main categories of multimedia services: Real-time (RT) and NonReal Time(NRT) services. RT services areeither delay-sensitive (e.g. VoIP) or loss-sensitive (e.g. buffered streaming) or both(e.g.video conferencing). Best effort services, anexample of NRT services, have higher delay requirements and spare resourcesare allocated to them. Dynamic subcarrier allocation, which is dependent on Channel Quality Information (CQI), has been broadly investigated for single carrier and multicarrier wireless networks in [1-6] and [12-14]. Maximising system throughput sometimes resultsin unfair allocation to the users located far from the Base Station (BS) or the users suffering from poor channel condition. Therefore a sufficient trade-off between throughput and fairness is essential. In [5], time, frequency and multiuser diversity in OFDM networks that lead to considerable efficiency have been discussed. Furthermore, cross-layer optimization based on utility function has also been introduced. The scarce bandwidth, fading channels and tough QoS requirements of users make resource allocation a demanding problem.

\subsection{Related Work}

For different service demands, a number of scheduling plans have been suggested.Three singlecarrier packet scheduling algorithms such as Round Robin(RR), Maximum Rate(Max-Rate) andProportional Fair (PF) have been investigated in[7],[8] and [9].MLWDF and Exponential Rule (EXP) were developed in [6] and [10] considering packet delay in Guaranteed Bit Rate(GBR) services over Code Division Multiple Access-High Data Rate (CDMA-HDR) systems.Channel-Dependent Earliest Due Dead line (CD-EDD) [11] algorithm in a mobile cellular system has been developed to study different delay requirements in GBRservices.In [6], M-LWDF scheduling employs both channel and delay information to prevent long queuing delay. In [12] and [13], the Max Delay Unit (MDU)scheduling scheme has been proposed in OFDM networks, and its performance has been compared withthe performance of MLDWF in [14]. In [15], LOG-MLWDF has been proposed and its performance has been investigated and compared withprevious methods proposed forOFDM networks.

Performance of MLWDF has been investigated in downlink 3GPP LTE system in [16, 17]. In this paper, three methods of packet scheduling will be investigated such as MDU, MLWDF and LOGMLWDF suggested for 3GPP LTEsystem.

The rest of this paper is organized as follows.The formulation of the problem and packet scheduling algorithms are given in Section 2.The simulation environment and results are discussed in Section 3 and Section 4 respectively. Finally, Section 5 contains the conclusions.

\section{SYSTEMMODEL AND PROBLEM FORMULATION}

System model in 3GPP LTE downlink and packet scheduling algorithms have been described in this section.

\subsection{System Model}

In 3GPP LTE system a pair of Resource Blocks (RBs) called Physical Resource Block (PRB), consisting of frequency and time domain resources,are assigned to users based on packet scheduling algorithms. The bandwidth of each $\mathrm{RB}$ is $180 \mathrm{kHz}$ in frequency domain which is 
composed of 12 sub-carriers. The duration of a RB is 0.5 msand consists of 7 OFDM symbols when normal prefix is used. Packet scheduling is carried out at eNodeB in $1 \mathrm{~ms}$ intervals and two consecutiveRBs are allocated to a user in that duration. At each time interval,users transmit their Channel State Information (CSI) to eNodeB within a RB.In this work, it has been assumed that the CSI report is based on the calculated SNR (Signal to Noise Ratio) value of the subcarrier at the central frequency of each RB, and assumed to be free of errors. All subcarriers of a RB are assumed to have the same SNR. According to the reported SNR, the eNodeBperforms scheduling for all users. In this paper, it has been assumed that all usershave buffers of infinite size in which packets are queued for transmission on a first in first out (FIFO) basis. The selection of the user (at each time slot and on each RB) is based on reported SNR and the priority (delay) of the user. Utility functions are used for selection of users based on SNR and delay. Figure 1 presents the architecture of the proposed scheduling schemes. The number of bits in two subsequentRBs is determined based on reported downlink SNR. In[18],the number of bits per symbol of user $i$ at time $\mathrm{t}$ on a subcarrier within $R B_{j}\left(\right.$ nbits $_{i, j}(t) /$ symbol)is used to compute the data rate of the user at time t. $d r_{i}(t)$,iscalculated according to thefollowingformula:

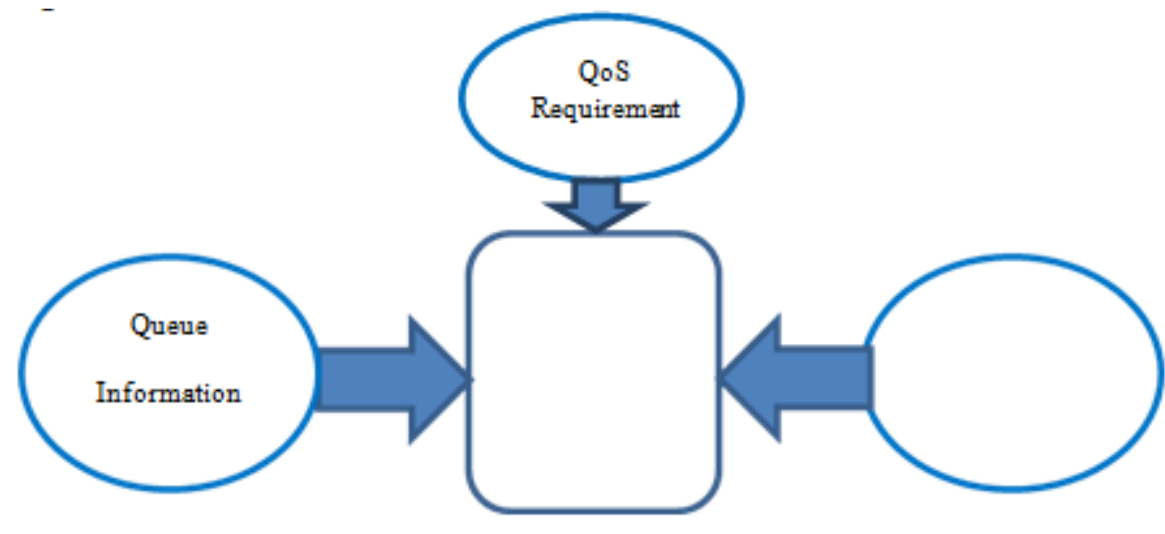

Figure1. Architecture of the proposed algorithms

$$
d r_{i}(t)=\frac{\text { nbits }_{i, j}(t)}{\text { symbol }} * \frac{\text { nsymbols }}{\text { slot }} * \frac{\text { nslots }}{T T I} * \frac{n s c}{R B}
$$

Where

$\frac{n s l o t s}{T T I}$ is the number of slots per TTI,

$\frac{n s c}{R B}$ is the number of subcarriers per $\mathrm{RB}$.

In Table1, minimum instantaneousdownlink SNR values and the related data rates have been given. User's priority is decided by packet scheduler based on channel condition, HOL(Head OfLine) packet delays and quality of service, etc. HOL packet delay is calculated by taking the 
International Journal of Wireless \& Mobile Networks (IJWMN) Vol. 7, No. 5, October 2015

difference of the current and arriving time of a packet. If the HOL delay exceeds a specific threshold then the packets will be discarded.

Table1. Immediate downlink SNR and data rate

\begin{tabular}{|l|l|l|}
\hline $\begin{array}{l}\text { MinimumInstantaneous } \\
\text { Downlink SNR } \\
\text { Value(dB) }\end{array}$ & $\begin{array}{l}\text { Modulation and } \\
\text { Coding }\end{array}$ & $\begin{array}{l}\text { Data } \\
\text { Rate(kbps) }\end{array}$ \\
\hline 1.7 & QPSK(1/2) & 168 \\
\hline 3.7 & QPSK(2/3) & 224 \\
\hline 4.5 & QPSK(3/4) & 252 \\
\hline 7.2 & $16 \operatorname{QAM}(1 / 2)$ & 336 \\
\hline 9.5 & $16 \operatorname{QAM}(2 / 3)$ & 448 \\
\hline 10.7 & $16 \mathrm{QAM}(3 / 4)$ & 504 \\
\hline 14.8 & $64 \mathrm{QAM}(2 / 3)$ & 672 \\
\hline 16.1 & $64 \operatorname{QAM}(3 / 4)$ & 756 \\
\hline
\end{tabular}

\subsection{Packet Scheduling Algorithms and Problem Formulation}

In this section, the mathematical model for MDU, M-LWDF and LOG-MLWDF scheduling algorithms are described. MDU and LOG-MLWDF have been described in [12] and [15] for OFDM networks and are suitable candidates to be used in 3GPP LTE networks.Model of the system includes one BS and M users. A multipath fading channel is assumed to be the model of wireless simulated channel. The formula given below explains the channel impulse model:

$h_{i}(t, \tau)=\sum_{k} \gamma_{k, i}(t) \delta\left(\tau-\tau_{k, i}\right)$.

Where $\tau_{k, i}$ is the delay of kth path and $\gamma_{k, i}(t)$ is the complex amplitude that is widestationary,narrowband and Gaussian random process. The paths are independent from each other. The channel frequency response is:

$H_{i}(f, t)=\sum_{k} \gamma_{k, i}(t) e^{-j 2 \pi f \tau_{k, i}}$

Signal to Noise Ratio (channel gain) for user i, is as follow:

$\rho_{i}(f)=\frac{\left|H_{i}(f)\right|^{2}}{N_{i}(f)}$ 
Where $N_{i}(f)$ is the noise power density.

Achievable rate of user $\mathrm{i}$ at frequency $\mathrm{f}, C_{i}(f)$, (assuming BER and power density to beconstant) is presented as follow:

$$
C_{i}(f)=\log _{2}\left(1+\frac{\beta p(f)\left|H_{i}(f)\right|^{2}}{N_{i}(f)}\right)=\log _{2}\left(1+\beta p(f) \rho_{i}(f)\right) \frac{\frac{b i t s}{s e c}}{H z}
$$

Where:

$$
\beta=\frac{1.5}{-\ln (5 B E R)}
$$

The throughput of user iis calculated as follows:

$r_{i}=\int_{D_{i}} C_{i}(f) \mathrm{df}$.

DSA(Dynamic Subcarrier Allocation)has been considered in this paper as the resource allocation scheme to dynamically allocate RBs to the users in order to maximize the average utility function as below:

$$
\operatorname{Max} \frac{1}{M} \sum_{i=1}^{M} U_{i}\left(r_{i}\right)
$$

Subject to:

$\bigcup_{i \in M} R B_{i}=[0, \beta]$,

$\operatorname{and} R B_{i} \cap R B_{j}=\emptyset, i \neq j, \forall i, j \in M$.

Assignment of one RB does not have any impact on the assignments of other RBs. Utility functions can be linear or nonlinear functions of the rate. DSA method can be used inthe following formula when the utility functions are linear:

$$
m(k, n)=\operatorname{argmax}_{i \in \mu}\left\{U_{i}^{\prime} C_{i}[k, n]\right\}
$$

Where $m(k, n)$ indicates that $R B_{k}$ is assigned to user $\mathrm{m}$ at time slot $\mathrm{n}$, and $C_{i}[k, n]$ is the feasible data rate for $R B_{k}$ at time slot $\mathrm{n}$, and it is totally calculated by CQI. With nonlinear utility function RBs cannot be allocated independently andin that case, DSA method would be very complicated. When the utility functions are concave, iterative algorithms are used.

\subsubsection{MDU Algorithm}

MDU[12] deploys the idea that utility function can be calculated based on average waiting time and it improves the quality of service idea. The MDU scheme formula is given below:

$\max _{R B^{n}, i \in A^{n}, s \in B^{n}} \sum_{i \in A^{n}, s \in B^{n}} \frac{\left|U_{i, s}^{\prime}\left(w_{i}\right)[n]\right|}{\lambda_{i, s}} r_{i}[n]$ 
Subject to the following conditions:

$\bigcup_{i \in A^{n}} R B_{i}^{n} \subseteq K$,

$R B_{i}^{n} \cap R B_{j}^{n}=\emptyset, i \neq j, \forall i, j \in A^{n}$.

$\lambda_{i, s}$ : Average arrival rate of user $i$ with the traffic type s,

$r_{i}[n]$ : Data transmission rate of user $i$ at time slot $n$,

$C_{i}[k, n]$ : Achievable transmission rate on subcarrier $R B_{k}$ for user $i$,

$R B_{i}^{n}$ : Set of subcarriers assigned to user $i$ at time slot $n$,

$A^{n}$ : Set of queues at time slot $n$,

$B^{n}:$ Set of the service types.

Three types of traffic such as voice, streaming and best effort have been considered in this work. Consecutive utility functions are used in MDU scheduling:

The marginal utility function for voice users is as below:

$\left|U_{i, v}^{\prime}\left(w_{i}\right)\right|=\left\{\begin{array}{c}w_{i}, w_{i} \leq 2.5 m s \\ w_{i}^{1.5}-2.5^{1.5}+2.5, w_{i} \geq 2.5 m s\end{array}\right.$

The marginal utility function for streaming users is given by:

$\left|U_{i, s}^{\prime}\left(w_{i}\right)\right|=\left\{\begin{array}{c}w_{i}^{0.6}, w_{i} \leq 5 m s \\ w_{i}-5+5^{0.6}, w_{i} \geq 5 m s\end{array}\right.$

The marginal utility function for best effort users is as follows:

$\left|U_{i, s}^{\prime}\left(w_{i}\right)\right|=\left\{\begin{array}{l}w_{i}^{0.5}, w_{i} \leq 5 m s \\ 5^{0.5}, w_{i} \geq 5 m s\end{array}\right.$

\subsubsection{MLWDF Algorithm}

The QoS requirements have been indicated in MLWDF[6] scheduling weights as below:

$$
a_{i}=-\frac{\log \delta_{i}}{T_{i}}
$$

$P_{r}\left\{W_{i}>T_{i}\right\} \leq \delta_{i}$

Where

$T_{i}$ :The delay threshold, 
$\delta_{i}:$ The maximum probability of exceeding $T_{i}$,

$W_{i}$ :The packet delay of user i.

MLWDF utility function is given by

$U_{i}=\gamma_{i} W_{i}(t) r_{i}(t)(11)$

Subjected to:

$\gamma_{i}=\frac{a_{i}}{\bar{r}_{i}}$

$W_{i}:$ The head of line packet delay for user i,

$r_{i}(\mathrm{n})$ : The channel rate of user $\mathrm{i}$,

$\overline{r_{l}}(\mathrm{n})$ : Average channel rate of user i.

When the precedence of service is high and the ongoing packet delay is considerable, the probability of obtaining service increases.

\subsubsection{LOG-MLWDF Algorithm}

LOG-MLWDF[15] is an extension of MLWDF algorithm in which logarithmic weight of the average delay has been added to MLWDF formula as below:

$$
U_{i}=\gamma_{i} r_{i}(t)\left(W_{i}+\log \left(\operatorname{Wave}_{i}(t)\right)\right)^{1.5}
$$

Where:

$\operatorname{Wave}(t)=\frac{1}{T} \sum_{i \in N} W_{i}(t)$.

Table 2.3GPP LTE Downlink system parameters

\begin{tabular}{|l|l|}
\hline Parameters & Values \\
\hline Carrier Frequency & $2 \mathrm{GHz}$ \\
\hline Bandwidth & $5 \mathrm{MHz}$ \\
\hline Number of Sub-carriers & 300 \\
\hline Number of RBs & 25 \\
\hline Number of Sub-carriers per RB & 12 \\
\hline Sub-Carrier Spacing & $15 \mathrm{kHz}$ \\
\hline
\end{tabular}


International Journal of Wireless \& Mobile Networks (IJWMN) Vol. 7, No. 5, October 2015

\begin{tabular}{|l|l|}
\hline Slot Duration & $0.5 \mathrm{~ms}$ \\
\hline Scheduling Time(TTI) & $1 \mathrm{~ms}$ \\
\hline Number of OFDM Symbols per Slot & 7 \\
\hline
\end{tabular}

\section{SIMULATIONENVIRONMENT}

In this paper a single cell of radius $150 \mathrm{~m}$ with downlink parameters in Table 1 has been modelled to study the performance of MDU, MLWDF and LOGMLWDF. The model which is described in previous section has been considered in this work. A cell of $5 \mathrm{MHz}$ bandwidth with $25 \mathrm{RBs}$ and 2 $\mathrm{GHz}$ carrier frequency is modelled. The parameters of the system are presented in Table 2.Two scenarios are considered in this research. In the first scenario, there are 40 voice and61 to 191 streaming users distributed uniformly within the simulation area.In the second scenario 1 to 36 best effort usershave been added to the network. Users are constantly moving between [1-100] $\mathrm{km} / \mathrm{h}$ speeds in random directions. To guarantee that the users always remain in the cell border's area, a wrap-around method is used. The SNR reports to the serving eNodeBare assumed to be instantaneous and free of delay and error. The buffer size for all the streaming and best effort users is assumed to be infinite.On-Off activity model has been chosen for modelling the voice source as conversational voice model. The average duration of $1 \mathrm{~s}$ and $1.35 \mathrm{~s}$ has been considered for exponentially distributed interval model of the voice. $32 \mathrm{kbps}$ digital voice coding is generated within each talk spurt (on).In Table 3, the parameters of simulated streaming video (with $128 \mathrm{kbps}$ rate) have been given. MLWDF, LOG-MLWDF and MDU methodshave been simulated for 3000 time slots (1ms each) usingMATLAB.When a packet's delay is more than the waiting time threshold, it is considered to bediscarded. The permissible waiting time of apacket in eNodeB buffer (considered as the threshold of HOL packet delay), is set to $20 \mathrm{~ms}, 10 \mathrm{~ms}$ and $100 \mathrm{~ms}$ for streaming, voice and best effort users respectively.

Table 3.Parameters of video streaming services [20]

\begin{tabular}{|c|c|c|}
\hline Information type & Distribution & $\begin{array}{l}\text { Distribution } \\
\text { Parameters }\end{array}$ \\
\hline $\begin{array}{l}\text { Inter-arrival time between the } \\
\text { beginning of successive } \\
\text { frames }\end{array}$ & $\begin{array}{l}\text { Deterministic } \\
\text { (Based on 20fps) }\end{array}$ & $50 \mathrm{~ms}$ \\
\hline $\begin{array}{l}\text { Number of packets(slices) in a } \\
\text { frame }\end{array}$ & Deterministic & 8 \\
\hline packets(slice) size & $\begin{array}{l}\text { Truncated } \\
\text { Pareto } \\
\text { (Truncated } \\
\text { Pareto } \\
\text { (Mean=50bytes, } \\
\text { max=125bytes) }\end{array}$ & $\begin{array}{c}\mathrm{K}=40 \text { bytes } \\
\quad \alpha=1.2\end{array}$ \\
\hline $\begin{array}{l}\text { Inter-arrival time between } \\
\text { packets(slices) in a frame }\end{array}$ & $\begin{array}{l}\text { Truncated Pareto } \\
(\text { Mean }=6 \mathrm{~ms}, \\
\operatorname{Max}=12.5 \mathrm{~ms})\end{array}$ & $\begin{array}{r}\mathrm{K}=2.5 \mathrm{~ms} \\
\quad \alpha=1.2\end{array}$ \\
\hline
\end{tabular}


Path loss, shadowing and Rayleigh fading have been considered forcombined path loss and shadowing model [19]. BER is assumed to be $10^{-6}$ for this paper.

To evaluate the performance of packet scheduling algorithms,system throughput, Packet Loss Ratio (PLR) and fairness have been used as performance metrics. Acomparison among thealgorithms is made based on these metrics. Throughput of the system is defined as the size of correctly received packets (in bits) during thesimulation time.The formula is as below:

averagethroughput $_{i}=\frac{1}{T} \sum_{i=1}^{N} \sum_{t=1}^{T}$ ptransmit $_{i}(t)$

Where $\mathrm{T}$ is total simulation time for user $\mathrm{i}, \mathrm{N}$ is number of users in service time and ptransmit $_{i}$ is total size of correctly received packets.PLR is defined as the ratio of total size of discarded packet to the total size of all packets that were received in the buffer of eNodeB during the interval. PLR of a system should be below thethreshold value to cover the QoS needs of a service. It is calculated according to following equation:

$$
P L R=\frac{\sum_{i=1}^{N} \sum_{t=1}^{T} \operatorname{pdiscard}_{i}(t)}{\sum_{i=1}^{N} \sum_{t=1}^{T} \operatorname{psize}_{i}(t)}
$$

Where, $\operatorname{pdiscard}_{i}(t)$ is the total size of discarded packets(in bits)of user $\mathrm{i}$ in the simulation time, $\operatorname{psize}_{i}(t)$ is the total size of all packets(in bits) that reaches the eNodeB buffer of user $i$ at simulation time T.N is total number of users and T is the simulation time.

Fairness is defined here as the difference between the most and least transmitted packets of two users divided by the total size of all packets that have arrived to the buffer of eNodeB. The formula is as below:

$$
\text { fainess }=1-\frac{\text { ptotaltransmit }_{\text {max }}-\text { ptotaltransmit }_{\text {min }}}{\sum_{i=1}^{N} \sum_{t=1}^{T} p \operatorname{size}_{i}(t)}
$$

In table 4, Guaranteed Bit Rate(GBR) and Non-GBR(NGBR) services and their different level of QoS requirements has been presented.

\begin{tabular}{|c|c|c|c|c|c|}
\hline & $\begin{array}{l}\text { Resource } \\
\text { Type }\end{array}$ & Priority & $\begin{array}{l}\text { Packet } \\
\text { Delay } \\
\text { Budget }\end{array}$ & $\begin{array}{l}\text { Packet Error } \\
\text { LossRate }\end{array}$ & Example Services \\
\hline 1 & \multirow{4}{*}{ GBR } & 2 & $100 \mathrm{~ms}$ & $10^{-2}$ & Conversational Voice \\
\hline 2 & & 4 & $150 \mathrm{~ms}$ & $10^{-3}$ & $\begin{array}{l}\text { Conversational Video(Live } \\
\text { Streaming) }\end{array}$ \\
\hline 3 & & 3 & $50 \mathrm{~ms}$ & $10^{-3}$ & Real Time Gaming \\
\hline 4 & & 5 & $300 \mathrm{~ms}$ & $10^{-6}$ & $\begin{array}{l}\text { None-Conversational Video } \\
\text { (Buffered Streaming) }\end{array}$ \\
\hline 5 & Non- & 1 & $100 \mathrm{~ms}$ & $10^{-6}$ & IMS Signal \\
\hline
\end{tabular}

Table 4.Standardized QoS Class Identifiers (QCIs)[21] 
International Journal of Wireless \& Mobile Networks (IJWMN) Vol. 7, No. 5, October 2015

\begin{tabular}{|c|c|c|c|c|c|}
\hline 6 & GBR & 6 & $300 \mathrm{~ms}$ & $10^{-6}$ & $\begin{array}{l}\text { Video } \\
\text { Streaming), } \quad \text { TCP-based } \\
\text { (e.g. email, chat..) }\end{array}$ \\
\hline 7 & & 7 & $100 \mathrm{~ms}$ & $10^{-3}$ & $\begin{array}{l}\text { Voice, Video (Live } \\
\text { Streaming),Interactive } \\
\text { Gaming }\end{array}$ \\
\hline 8 & & 8 & $300 \mathrm{~ms}$ & $10^{-6}$ & $\begin{array}{l}\text { Video(Buffered } \\
\text { Streaming)TCP-based (e.g. }\end{array}$ \\
\hline 9 & & 9 & & & www,e-mail, chat..) \\
\hline
\end{tabular}

\section{SIMULATION RESULTS}

In this section, the simulation results are analysed. Figures 2 to 6 are related tothe first scenario in which there are no best effort users in the network. Figure2 and Figure 3 show the system throughput graphs for streaming and voice users of the three packet scheduling algorithms. Throughput of streaming users are the same in MLWDF and LOG-MLWDF algorithms (as seen in Figure 2), whereas for voice users, MLWDF has better throughput performance compared with LOG-MLWDF and MDU methods (as shown in Figure 3).

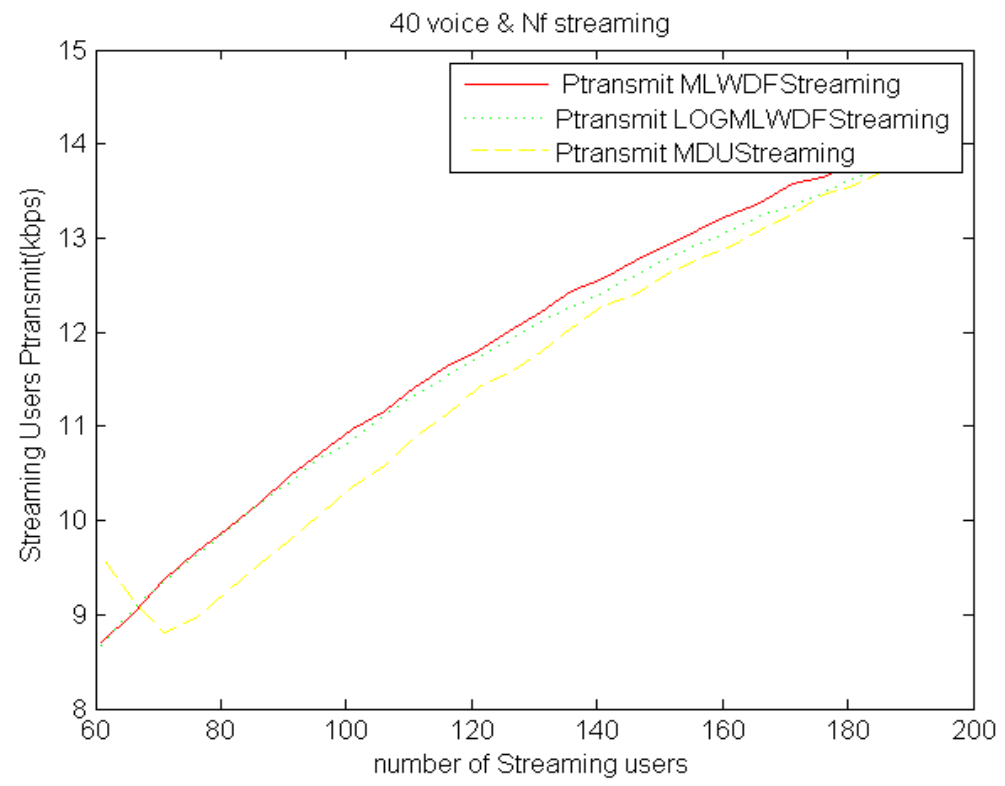

Figure 2.System throughput for streaming users vs. numberof steaming users 
International Journal of Wireless \& Mobile Networks (IJWMN) Vol. 7, No. 5, October 2015

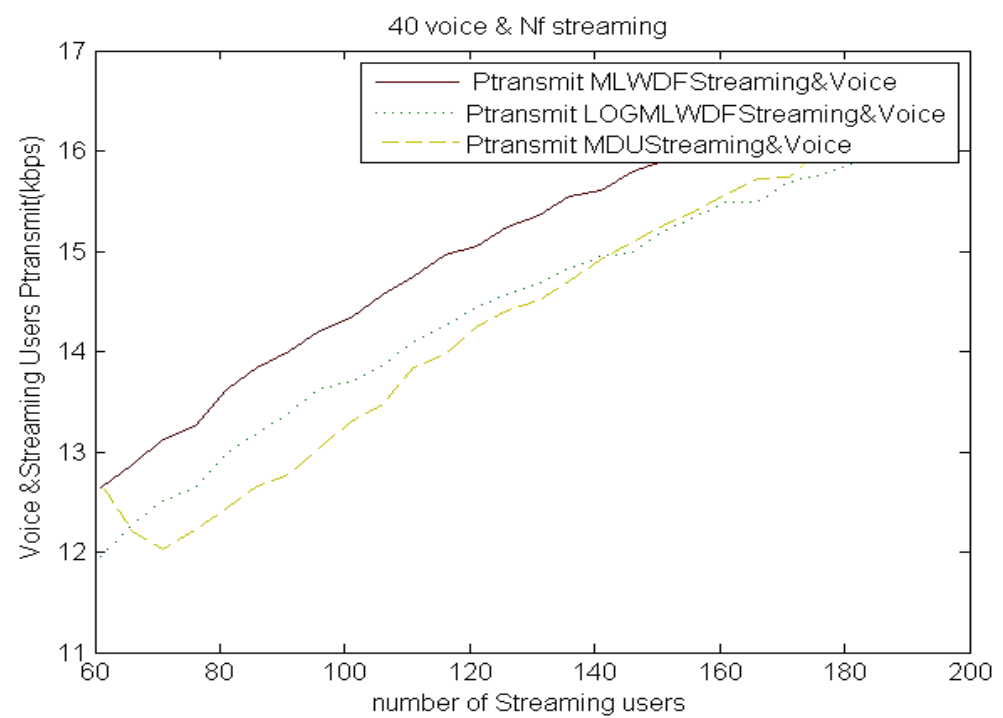

Figure 3.System throughput for streaming \&voice users vs. number of streaming users

Figure 4 and 5showPLR performance for the three algorithms. It can be observed from Figure 4 that for all streaming users, LOG-MLWDF has satisfied thePLR threshold of $10^{-3}$ [21]. With increasing number of streaming users, MLWDF and MDU have greaterPLR than LOG-MLWDF for streaming users. For voice users, LOG-MLWDF has greaterPLR than the other twoalgorithms (Figure 5) even thoughthe PLR is still below the permissible threshold for conversational voice of $10^{-2}$ [21]. The MLWDF followed by LOG-MLWDF have the highestPLR for streaming and voice users.

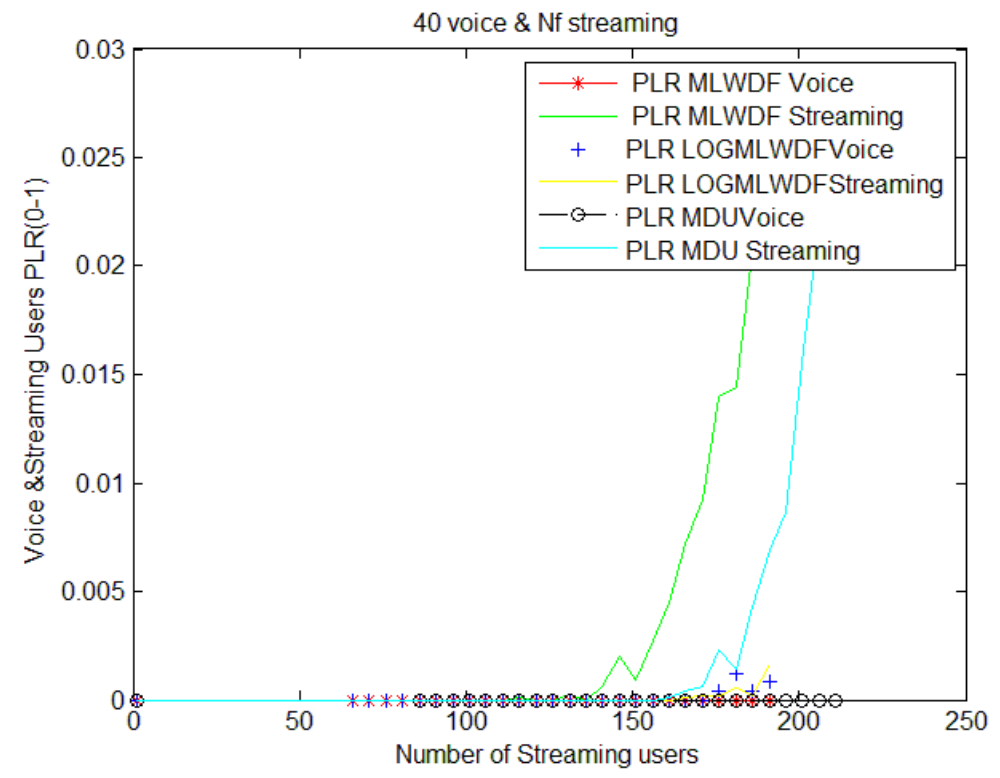

Figure 4. Packet Loss Ratio vs. number of streaming users 
International Journal of Wireless \& Mobile Networks (IJWMN) Vol. 7, No. 5, October 2015

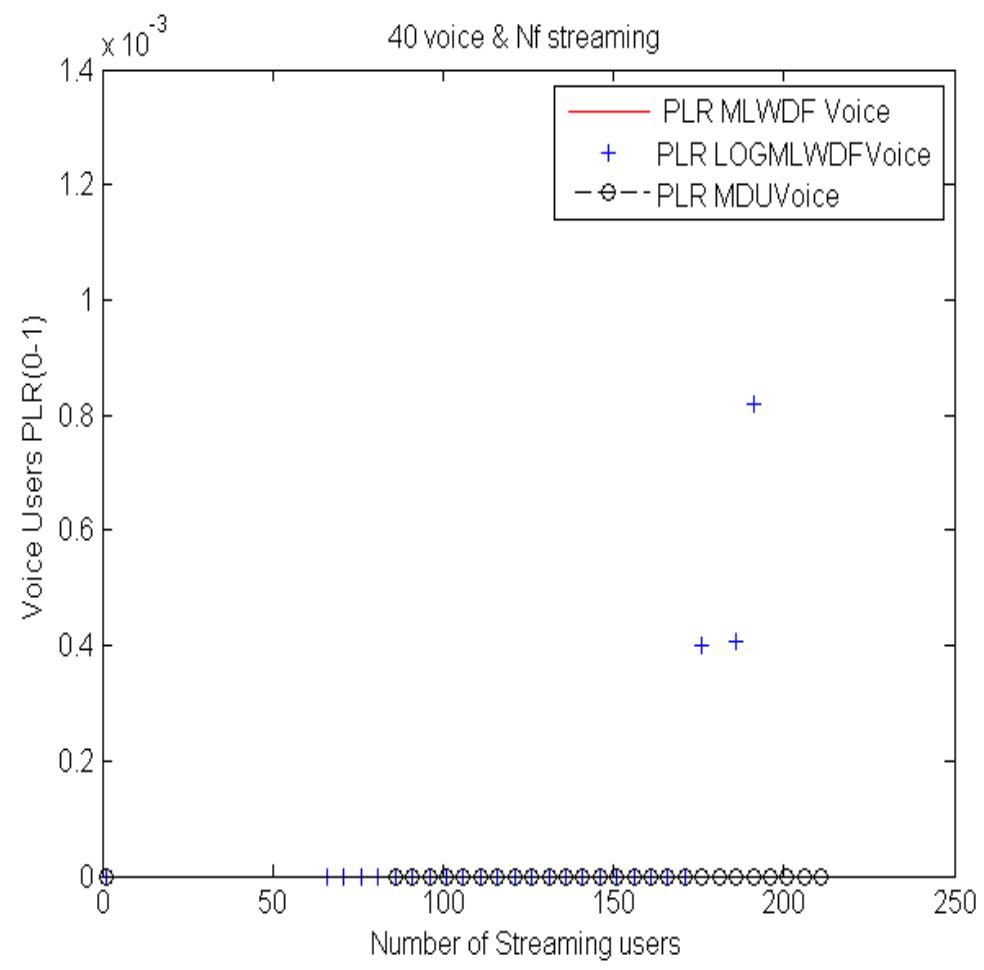

Figure5. Packet Loss Ratio vs. number of streaming users

In Figure 6 the fairness of the three algorithms has been compared. LOG-MLWDF has the worst fairness performance among the three methods. The fairness performance of MLWDF is slightly better than MDU algorithm when the number of users is low but it is slightly lower than MDU algorithm when the number of users is high. 
International Journal of Wireless \& Mobile Networks (IJWMN) Vol. 7, No. 5, October 2015

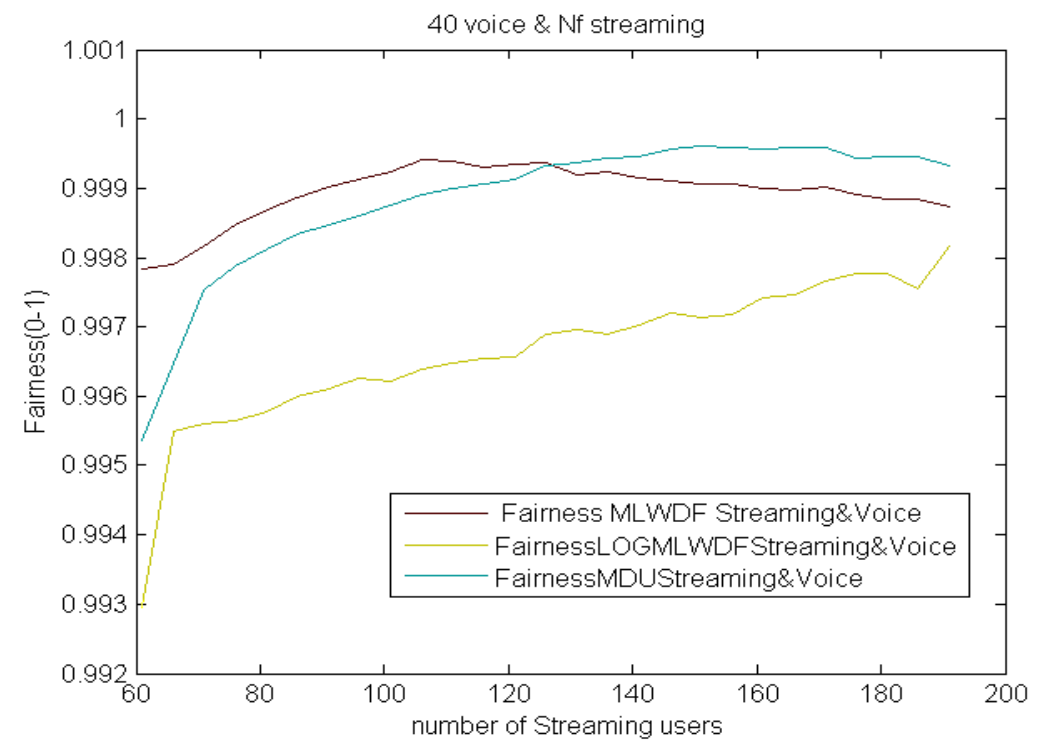

Figure 6.Fairness vs. number of streaming users

Considering the second scenario in which best effort users are added to the previous network simulation. The simulation results are given in Figure7 and Figure 8.From the percentile delay and throughput perspective of best effort users, it can be seen that LOG-MLWDF outperforms the previous algorithms.

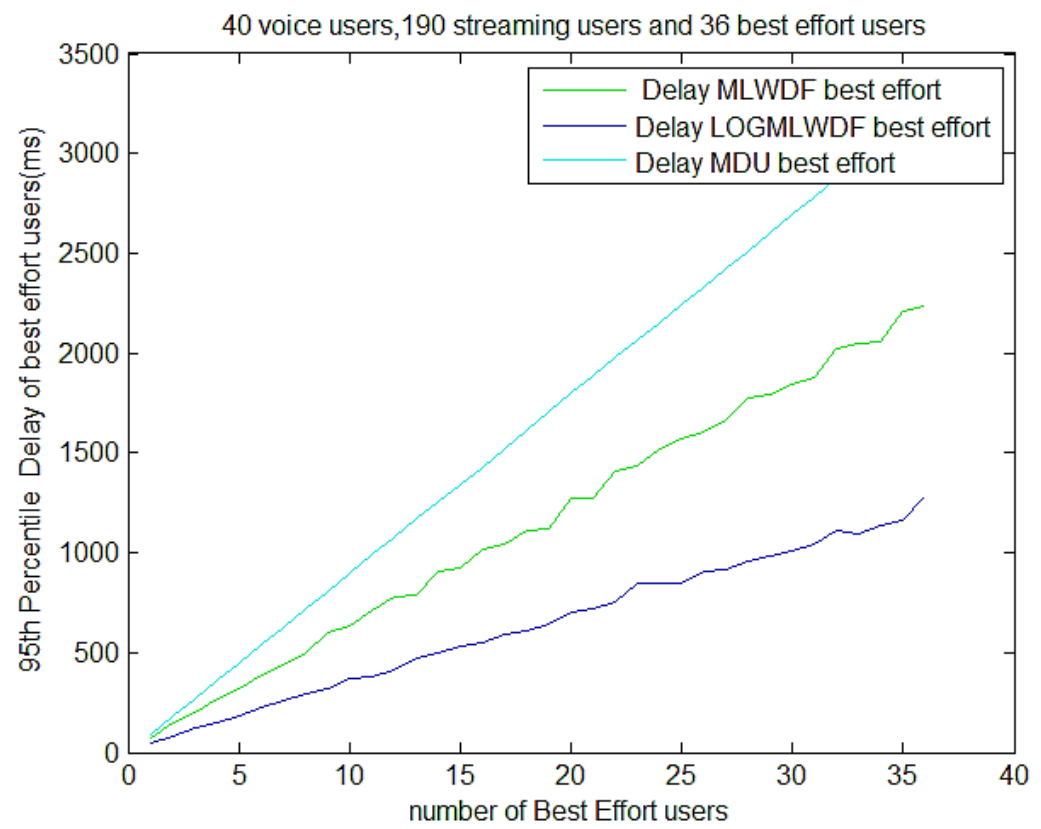

Figure 7.95 percentile delay of best effort users vs. number of users 
International Journal of Wireless \& Mobile Networks (IJWMN) Vol. 7, No. 5, October 2015

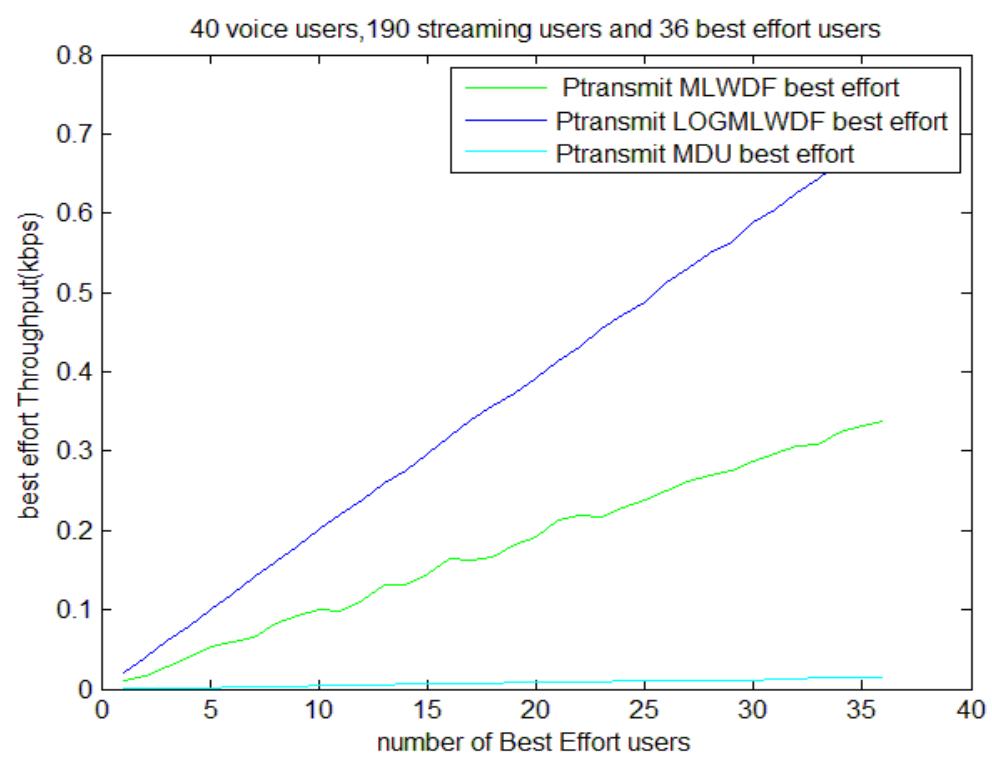

Figure8. Best effort throughput vs.number of users

In Figure 9, the three algorithms have been compared according to the fairness metric. It can be seen that LOG-MLWDF outperforms the other two algorithms and MLWDF has the least fairness compared with other algorithms.

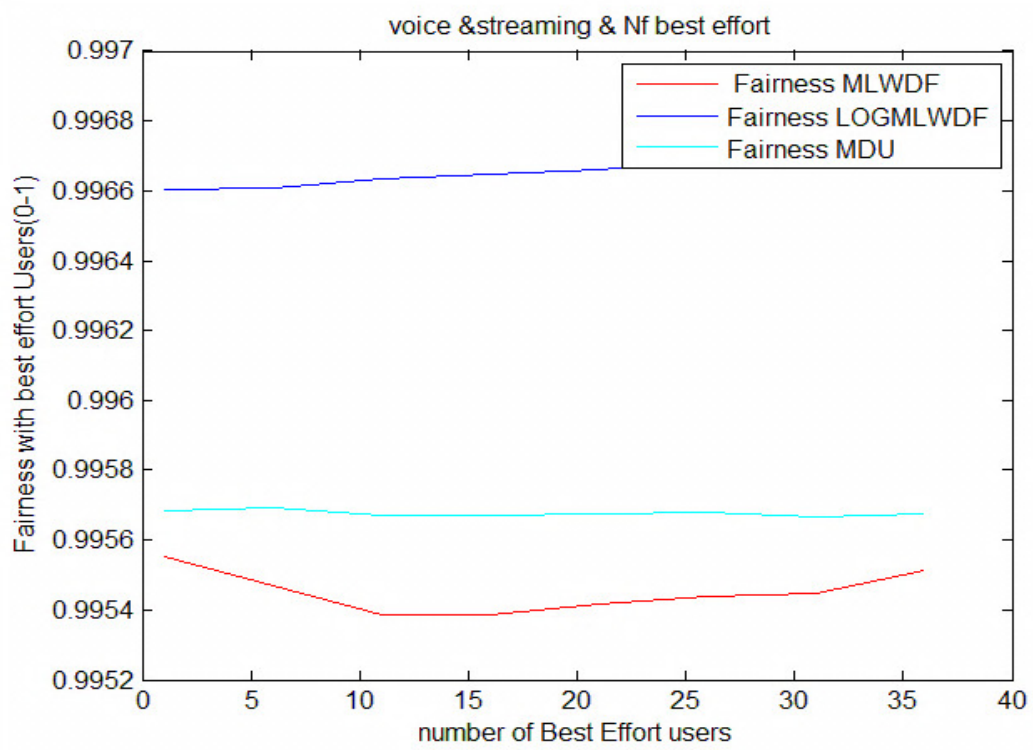

Figure 9. Fairness vs. number of users 


\section{CONCLUSIONS}

This paper investigates the resource management of three scheduling algorithms in a downlink 3GPP LTE system. A comparison between well-known algorithms MDU,MLWDF and LOGMLWDF has been made considering two scenarios. This paper also analyses the strengths and weaknesses of LOG-MLWDF compared with the other two algorithms. In the first scenario, LOG-MLWDF shows better PLR performance for streaming users and its PLR performance remains below the threshold value for voice users. With the addition of best effort users it is apparent that LOG-MLWDF outperforms the two other algorithms in terms of 95 percentile delay, fairness of the wireless network and throughput for best effort users. In future work different scheduling scheme with the concept of Hybrid Automatic Repeat Request (HARQ) with different simulation time and more scenarios will be investigated.

\section{REFERENCES}

[1] C.Y. Wong, R. S. Cheng, K. Ben Letaief, and R. D. Murch, "Multiuser OFDM with adaptive subcarrier, bit, and power allocation," IEEE JSAC, vol.17,no. 10, pp.1747-1758, Oct. 1999.

[2] K. Navaie, D. Y. Montuno, and Y. Q. Zhao , "Fairness of resource allocation in cellular networks: A survey," Resource Allocation in Next Generation Wireless Networks, W. Li and Y. Pan, Eds. Nova Science Publishers, 2005.

[3] C.U. Saraydar, N. Mandayam, and D. Goodman, "Pricing and power control in a multi cell wireless data network," IEEE JSAC, vol. 19, no. 10, pp. 1883-1892, Oct. 2001.

[4] K. Navaie and H. Yanikomeroglu, "Optimal Downlink Resource Allocation for Non-real time Traffic in Cellular CDMA/TDMA Networks,” IEEE Communications Letters, vol. 10, pp. 278-280, April 2006.

[5] G. Song and Y. (G). Li, "Cross Layer Optimization for OFDM Wireless Network-part I and part II," IEEE Trans. Wireless Communication, vol. 4, March 2005.

[6] Mathew Andrews, Rajiv Vijayakumar, "Providing Quality of Service over a Shared Wireless Link," IEEE Communication Magazine, Feb. 2001.

[7] E. Dahlman, S. Parkvall, J. Skold, and P. Beming, "3G Evolution: HSPA and LTE for Mobile Broadband,'First ed. Elsevier Ltd., 2007.

[8] B.S. Tsybakov,"File Transmission over Wireless Fast Fading Downlink," IEE Transaction on information Theory, vol. 48, no. 8, pp. 2323-2337, 2002.

[9] A. Jalali, et al. , "Data Throughput of CDMA-HDR a High Efficiency-High Data Rate Personal Communication Wireless System," in IEEE 51st Vehicular Technology Conference Proceedings, 2000, pp.1854-1858.

[10] S. Shakkotai and A. L. Stolyar, "Scheduling Algorithms for a Mixture of Real-Time and Non-RealTime Data in HDR," in Teletraffic Science and Engineering, pp. 793-804, 2001.

[11] A. K. F. Khattab and K. M. F. Elsayed, "Channel-quality dependent earliest deadline due fair scheduling schemes for wireless multimedia networks," in 7thACM International Symposium on Modelling, Analysis and Simulation of Wireless and Mobile Systems, 2004, pp. 1-8.

[12] G. Song, Y.(G). Li, Leonard J. Cimini, Jr. HaitareZheng, "Joint Channel Aware and Queue Aware Data Scheduling in Multiple Shared Wireless Channels," WCNC 2004/IEEE Communication Society.

[13] Liu Zhe, Xinglin Wang, "A Simplified Layered QoS Scheduling Scheme in OFDM Networks," Vehicular Technology Conference, Fall 2007.

[14] G. Song, Y. (G). Li, "Utility Based Resource Allocation and Scheduling in OFDM Based Wireless Broadband Networks," IEEE Communication Magazine, December 2005.

[15] R. Heidari, M. Mehrjoo, "Delay and Rate based Multichannel Scheduling for Heterogeneous Traffic", IEEE CNDS Conference, Feb. 2011. 
International Journal of Wireless \& Mobile Networks (IJWMN) Vol. 7, No. 5, October 2015

[16] R. Basukala, H.A. MohdRamli, "Performance Analysis of EXP/PF and M-LWDF in Downlink 3GPP LTE System", IEEE AHICI Conference, Nov. 2009.

[17] H. AdibahMohdRamli, RiyajBasukala, "Performance of Well Known Packet Scheduling Algorithms in the Downlink 3GPP LTE System", IEEE Communication MICC Conference, Dec.2009.

[18] X. Qiu and K. Chawla, "On the Performance of Adaptive Modulation in Cellular Systems," IEEE Transactions on Communications,vol. 47, 1999, pp. 884-895.

[19] Andrea Gold Smith, "Wireless Communication", Cambridge University Press, 2005.

[20] 3GPP2," CDMA 2000 Evaluation Methodology," December 2004.

[21] 3GPP (2009b) TS 23.203 (V9.3.0) "Policy and Charging Control Architecture", Release 9. 